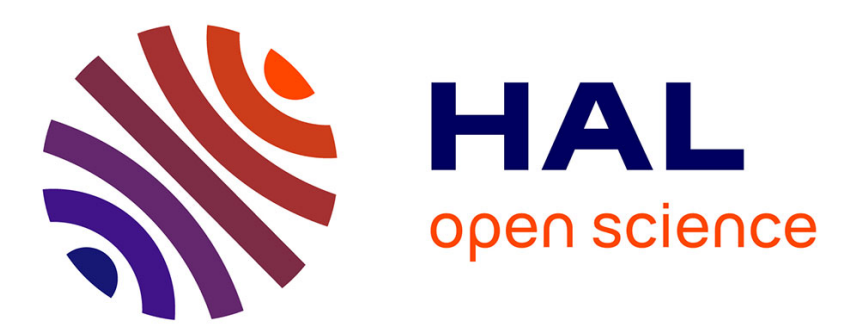

\title{
Transmit Beamforming Analysis for MIMO Systems in Indoor Residential Environment Based on 3D Ray Tracing
}

\author{
A Bouhlel, V Guillet, Ghais El Zein, Gheorghe Zaharia
}

\section{- To cite this version:}

A Bouhlel, V Guillet, Ghais El Zein, Gheorghe Zaharia. Transmit Beamforming Analysis for MIMO Systems in Indoor Residential Environment Based on 3D Ray Tracing. Wireless Personal Communications, 2015, 82 (1), pp.509-531. 10.1007/s11277-014-2238-5 . hal-01100992

\section{HAL Id: hal-01100992 \\ https://hal.science/hal-01100992}

Submitted on 7 Jan 2015

HAL is a multi-disciplinary open access archive for the deposit and dissemination of scientific research documents, whether they are published or not. The documents may come from teaching and research institutions in France or abroad, or from public or private research centers.
L'archive ouverte pluridisciplinaire HAL, est destinée au dépôt et à la diffusion de documents scientifiques de niveau recherche, publiés ou non, émanant des établissements d'enseignement et de recherche français ou étrangers, des laboratoires publics ou privés. 


\title{
Transmit Beamforming Analysis for MIMO Systems in Indoor Residential Environment Based on 3D Ray Tracing
}

\author{
A. BOUHLEL ${ }^{1}$, V. GUILLET ${ }^{1}$, G. EL ZEIN ${ }^{2}$ and G. ZAHARIA ${ }^{2}$ \\ 1 Orange Labs, Wireless Engineering and Propagation, 1 Rue Louis et Maurice de Broglie, Bâtiment \\ 11, 90000 Belfort, France \\ 2 Institute of Electronics and Telecommunications of Rennes, IETR, UMR CNRS 6164, 20 Avenue des \\ Buttes de Coësmes, CS 70839, 35708 Rennes Cedex 7, France
}

E-mail: ghais.el-zein@insa-rennes.fr, Tel: +33 (0)2 232386 04, Fax: +33 (0)2 23238439

\begin{abstract}
In this paper, an evaluation of transmit beamforming (TxBF) contribution is analyzed in the context of an indoor residential environment. Using an optimized 3D ray tracing tool, the multipleinput multiple-output (MIMO) propagation channel is simulated. The algorithm of singular value decomposition is applied and a new transmission scheme is considered. Comparisons of cases with/without TxBF are realized by analyzing the received power, the signal to noise ratio (SNR) of each spatial stream and the channel capacity. It is shown that the TxBF capacity gain increases when the MIMO channel correlation at the transmit side reaches high values, especially when only the dominant eigen mode is selected. The main result concerns the antenna spacing: a judicious choice of the transmitting antenna spacing can improve the effective transmission range by more than $45 \%$ in non-line of sight (NLOS) conditions. Several MIMO configurations (number of antennas and spatial streams) are also compared to determine the relevance of a possible activation of TxBF. The interference influence is studied and it is shown that under some conditions, TxBF improves the signal to interference ratio (SIR).
\end{abstract}

Key words: beamforming, MIMO, indoor, radio propagation, correlation.

\section{Introduction}

Multipath is considered to be the major problem in basic wireless systems. It causes fading as radio waves travel over multiple paths and interfere with each other when they arrive at the receiver. Preprocessing the signal at the transmitter, using a technique known as transmit beamforming (TxBF), can overcome multipath effects to improve link throughput and robustness.

Beamforming is an optional feature of $802.11 \mathrm{n} / \mathrm{ac}$ standards but there is a growing industry demand for it in various wireless applications. It is a natural extension of the physical layer that has multiple spatial streams and antennas at both ends. In the case of TxBF, by controlling the power and the phase of the transmitted signals, it is possible to shape the effective radiation pattern of the antennas pointing towards the direction of the dominant path to improve the received power and hence the signal to noise ratio $(S N R)$. TxBF studied in this paper is used to extend the effective transmission range. It creates more robust coverage for 802.11n/ac systems. Various techniques may be used to implement TxBF such as phased antenna arrays (direction based beamformers), codes book (for LTE), dominant eigen mode selection (single beamforming technique with one antenna weighting vector and that carries only one spatial stream [1]), and the more general SVD-MIMO (multiple eigen beamforming with one precoding matrix that carries multiple spatial streams such as in $802.11 \mathrm{n} / \mathrm{ac}$ for example) which is studied in this paper. 
Efficient steering of individual streams in such a system provides overall gain. Beamforming gain consists of:

- an increased average received power compared with omnidirectional reception/transmission.

- a diversity gain: through an optimized recombination of multipath that reduces the signals fading.

In the literature, TxBF has been studied in order to highlight its contribution compared with the use of a simple MIMO system using spatial multiplexing only, like standardized for 802.11n for example.

Most of the publications studied outdoor [2] or outdoor-indoor [3] environments. They are based either on measurements made with equipment that implements TxBF or on a purely theoretical approach. Most of the results concern the dominant eigen mode TxBF, but there are few results concerning the more general SVD-MIMO systems with multiple spatial streams [4] [5], and they rarely concern typical European residential environments and European building materials. In fact, studies have analyzed several MIMO configurations in order to conclude on the relevance of a possible use of beamforming. This option requires the knowledge of the MIMO propagation channel between the transmitter and the receiver. This implies frame exchanges between transmitter and receiver and an overhead in the frame control. For instance, in [6], it was shown that for small values of SNR (or high interference level) the dominant eigen mode BF schemes can perform close to the MIMO system with singular value decomposition in terms of spectral efficiency, and can even outperform a MIMO system with Channel State Information (CSI) only at the receiver.

Other studies have focused on the comparison between introducing the dominant eigen mode and transmit diversity as two complementary techniques for using multiple antennas in the forward link of a cellular communication system [7]. It was shown that, under ideal conditions (uncorrelated fading), transmit diversity has an advantage over a beamforming system. However, under handoff conditions, the beamforming system has both array gain and diversity, which improves its performance relative to transmit diversity.

The singular values decomposition (SVD), which is seen as one of the most relevant MIMO precoding techniques, is present in publications that address the topic of eigen beamforming with multiple streams. In fact, in [8]-[11], analyzes have focused on evaluating the impact of channel estimation error on the performance of MIMO system that implements SVD-MIMO technique. It was proved that in case of non-ideal channel knowledge and a limited accuracy in the channel matrix estimation, a reduced number of eigenmodes in the precoding process becomes an optimum and leads into an improved BER. A typical system employing SVD suffers capacity degradation when incorrect CSI is used to transmit data. Therefore, some papers propose a new linear processing architecture which reduces the effect of incorrect CSI at the transmitter.

Other approaches such as prototyping [12] have been also explored in order to assess the feasibility of an IEEE $802.11 \mathrm{n}$ transmit beamforming architecture before starting its mass production. But in this case, it is another study that tests the effect of the feedback delay for CSI. It does not exploit this realistic process to evaluate the added value of beamforming.

Thus, in the context of earlier studies, it was shown in [13] for example, that in the case of a single stream transported by a MIMO system with NTx transmit antennas and NRx receiving antennas $(N R x \times N T x$ MIMO), a significant antenna array gain $N R x$. NTx can be achieved in Line Of Sight (LOS) conditions i.e. in a fully correlated received signals. For multiple streams and using the SVD-MIMO technique, a theoretical bound of the gain was defined in the case of a rich scattering environment (Rayleigh fading). This bound is $(\sqrt{N R x}+\sqrt{N T x})^{2}$. Other works such as [14] have also analyzed the diversity gain for such 
channels and SVD-MIMO technique, and calculated that the achievable diversity order is $\left(N T x-N_{s s}+1\right) .\left(N R x-N_{s s}+1\right)$ for $N_{s s}$ spatial streams. So many aspects remain to develop, which are mainly:

- An evaluation of the antenna array gain and diversity gain in the case of a real indoor environment (LOS and NLOS) with non-ideal channel correlation properties.

- A practical study of the gains in the case of SVD-MIMO with multiple spatial streams rather than considering only the dominant mode.

Therefore, the results presented in this paper fall within the general framework of studying transmit beamforming. They reveal in particular what can bring the introduction of this option compared to the use of a simple MIMO system with spatial multiplexing. A typical indoor residential environment is considered for this work. The comparisons will be expressed as a function of several parameters: the power received by all the receiving antennas, the signal to noise ratio $(S N R)$ and the channel capacity. These different performance criteria will be considered in both configurations without beamforming (WO-BF) and with beamforming (W-BF) using the SVD-MIMO technique. The analysis will focus on the effect of TxBF on:

- Each spatial stream: the SNR of each spatial stream will be computed in W-BF and WOBF.

- The entire MIMO configuration: MIMO configuration is the pair (number of antennas, number of spatial streams).

Several MIMO configurations (number of antennas, number of spatial streams, spacing between antennas) are also compared to identify which one has the most important BF gain. An interference scheme will then be reviewed with a jammer link disturbing the desired signal. The aim is to investigate whether the introduction of BF can increase or reduce the effect of interference generated by an interfering link.

The rest of the paper is organized as follows. We introduce the channel model and the simulation environment in Section 2. In Section 3, the results of a comparative study of several performance criteria are presented and discussed. The received power, the SNR and the channel capacity are analyzed with and without transmit BF, as well as the BF gain expressed as a function of channel parameters (correlation and antenna spacing). In Section 4, we compare the signal to interference ratios (SIR). Finally, we draw some conclusions in Section 5.

\section{Channel Model and Simulation Environment}

\subsection{RAY TRACING SimUlation TOOL}

The results presented in this paper rely on a simulation approach. A 3D ray tracing model developed previously is used to have a realistic MIMO channel modeling [15] without any hypothesis on the channel correlation. This is our basis to construct the MIMO channel matrix used for beamforming performance analysis.

The ray tracing software calculates the ray paths between each transmitting and each receiving antenna. It is based on the geometrical optics (GO) and the uniform geometrical theory of diffraction (UTD). The propagation phenomena taken into account are combinations of multiple transmissions, reflections and diffractions. Thus, a detailed description of the 3D indoor environment is essential for a proper modeling of wave propagation. It requires taking 
into account the electromagnetic characteristics of walls, floor, ceiling, windows and doors, and some details about the furniture. Plane facets with 3D dimensions describe the simulated indoor environment. Each facet is affected to a material which is characterized by its permittivity, conductivity and thickness. The electromagnetic properties of various building materials have been estimated in a previous study over the 2-16 GHz frequency band [16]. Consequently, the software tool can operate over a large frequency range. As a result, for each radio link, the simulations provide a list of all the identified ray paths. Each ray is characterized by its complex propagation vector, absolute delay, angle of arrival (AoA) and angle of departure (AoD). It is therefore possible to easily estimate channel spatio-temporal characteristics such as delay spread and angular spread. Figure 1 shows the typical 3D indoor residential scene used to perform simulations. It is a typical and real middle sized apartment with a $12 \mathrm{~m} \times 7 \mathrm{~m}$ surface and European building materials and furniture. Two transmitter (Tx) locations have been considered with 25 receiver $(\mathrm{Rx})$ locations in LOS and NLOS. Both Tx and Rx antenna arrays are $1 \mathrm{~m}$ above the ground level. The ceiling is at $2.53 \mathrm{~m}$.

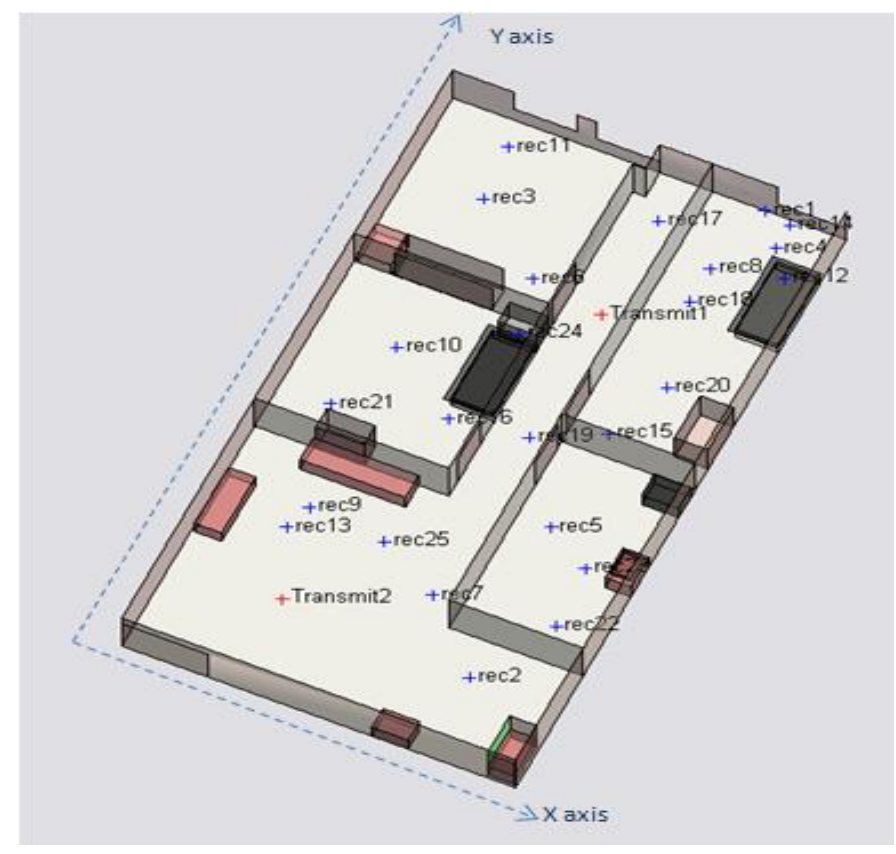

Figure 1. The indoor environment: 2 Tx positions (red) and $25 \mathrm{Rx}$ positions (blue).

\subsection{Simulations}

The realization of the MIMO channel simulations was conducted in three steps:

i) Ray Tracing

The ray tracing software is launched considering a transmitter and a receiver to trace all the rays between a source station located in the corridor (Transmit1) and a receiver placed in 25 positions distributed in different rooms of our indoor environment (Figure 1). A transmitter or a receiver consists of four omnidirectional vertically polarized dipole antennas arranged in form of a square (Figure 2).

This configuration allows analyzing several MIMO systems such as $4 \times 4,4 \times 3,4 \times 2,3$ $\times 4,3 \times 2,2 \times 2$, etc. No antenna coupling effects are considered in this study. For $4 \times 4$ 
MIMO, $4 \times 4 \times 25=400$ ray traces have been launched considering a single Tx location with 4 antennas and $25 \mathrm{Rx}$ with 4 antennas.

For each Rx location, 10 carrier frequencies of the considered OFDM signals with $2 \mathrm{MHz}$ spacing have been selected. They are distributed over a $20 \mathrm{MHz}$ bandwidth around the central frequency $5.5 \mathrm{GHz}$. In fact, not all the carriers have been processed since in indoor residential environment, channel correlation bandwidth is relatively high compared to the OFDM carrier spacing of a $802.11 \mathrm{n} / \mathrm{ac}$ signal: typical delay spread $\sigma$ measured in this environment [15] is between 5 and $20 \mathrm{~ns}$, so we can estimate the $90 \%$ correlation bandwidth $\mathrm{B}=50 / \sigma$ is between 1 and $4 \mathrm{MHz}$. The results have been then processed to compute the power of the rays for each one of the 10 selected carriers. Finally, 250 samples of the $4 \times 4$ MIMO channel were obtained.

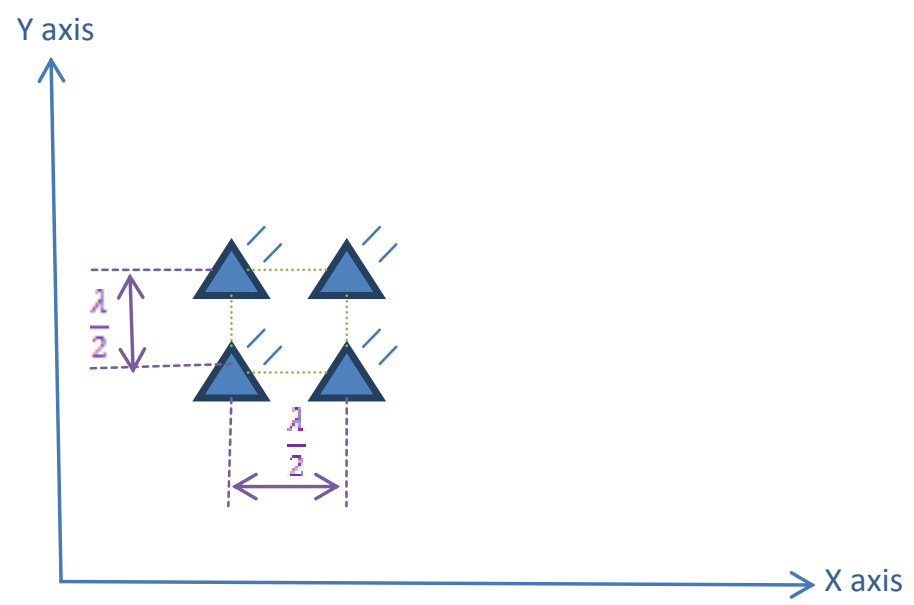

Figure 2. The antenna array.

To run all the simulations in a reasonable time (several hours for all), we have chosen the number of phenomena undergone by each ray. In order to obtain a tradeoff between reliability and computational time, our ray tracing tool was configured with 3 reflections, 1 diffraction and 5 transmissions: this choice is justified by the comparisons between simulations and measurements performed in this apartment [15].

\section{ii) Channel Matrix Calculation}

The ray tracing tool outputs are generated as files. In this stage, we extract data from these files to calculate the MIMO channel matrix. 250 matrices $(\mathrm{H})$ are then calculated $(25 \mathrm{Rx}$ locations and 10 carrier frequencies for each $\mathrm{Rx}$ location). In a real connection, this step corresponds to the channel estimation performed at the transmitter by exploiting the channel state information (CSI). It is followed by the decomposition of the matrix $\mathrm{H}$ in singular values.

\section{iii) Results Analysis}

This third part will be detailed in Sections 3 and 4 .

\subsection{SVD DECOMPOSITION}

Conceptually, the TxBF objective is to optimize the Tx antenna array pattern to maximize the power or the signal to noise ratio of the received signal for the desired user. Assuming that the channel matrix $\mathrm{H}$ is known, then, the optimal beamforming weights at the transmitter can be 
obtained using singular value decomposition (SVD) [17]. In SVD-based MIMO-OFDM communication systems, SVD of $2 \times 2$ (or $4 \times 4$ ) complex matrices are periodically computed for each one of the OFDM carrier frequency. A general channel model can be described by

$$
r_{W O-B F}=H X+n
$$

where $r_{W O-B F}$ is the received signal column vector, $X$ is the transmitted signal, $H$ a $N R x \times$ $N T x$ complex matrix for the MIMO channel for a single OFDM carrier and $n$ is a column vector of $N R x$ additive complex Gaussian noise with zero mean and equal variance for the independent real and imaginary components. It is also assumed that the noises of each receiving antenna are independent random variables.

Precoding consists in the spatial processing that occurs at the transmitter to maximize the signal power at the receiver input. The system requires the knowledge of the channel state information (CSI) at the transmitter [10], [11]. In this paper, perfect channel estimation is assumed.

For a system with $N T x$ transmitter antennas and $N R x$ receiver antennas, the singular value decomposition of a matrix $H$ is given by

$$
H=U^{0} S^{0} V^{0^{*}}
$$

where $U^{0}, V^{0}$ are respectively a $N R x \times N R x$ and $N T x \times N T x$ unitary matrices $\left(U^{0^{*}} U^{0}=\right.$ $I_{N R x \times N R x}$ and $V^{0^{*}} V^{0}=I_{N T x \times N T x}$ ) and (.)* means transpose-conjugate. $S^{0}$ is a $N R x \times N T x$ diagonal matrix with the square root of eigen values of $H^{*} H$, where $s_{i}$ are arranged in decreasing order. When NTx $<N R x, S^{0}$ can be written as

$$
S^{0}=\left(\begin{array}{cccc}
s_{1} & 0 & \cdots & 0 \\
0 & s_{2} & 0 & \vdots \\
\vdots & 0 & \ddots & \vdots \\
\vdots & \cdots & 0 & s_{N T x} \\
0 & \cdots & 0 & 0
\end{array}\right)
$$

The singular values $s_{i}$ are non-negative, ordered in decreasing order and defines the quality of the $i^{\text {th }}$ eigenmode. For example, to transmit $N_{s s}<N T x$ spatial streams on NTx antennas, only the first $N_{s s}$ columns of $V^{0}$ are considered to construct the precoding matrix $V$, corresponding to the main $N_{s s}$ eigen modes. Obviously, we need to have at least $N_{s s}$ transmitting and receiving antennas and at least $N_{s s}$ non-zero singular values. For that, the size of the precoding matrix $V$ becomes $N T x \times N_{s s}$. SVD-based precoding techniques make use of the fact that the matrix $V$ consisting of a number of columns of $V^{0}$ contains eigenvectors of $H^{*} H$, which correspond to the eigenmodes of the communication channel.

It can be written as $H V=U S$, where $U$ is the matrix formed with the $N_{s s}$ first columns of $U^{0}$ and $S$ is a $N_{s s} \times N_{s s}$ matrix. Note that $U$ and $V$ are not necessary unitary matrices: $U=I_{N S S \times N S S}$, but if $N s s<N R x$, then $U U^{*} \neq I_{N R x \times N R x}$.

The precoding technique transmits the matrix product $V X$. The received signal $r_{W-B F}$ is

$$
r_{W-B F}=H V X+n
$$




\section{Performance Optimization Using Beamforming}

In this section, the TxBF is quantified in terms of instantaneous received power (Section 3.1), SNR and channel capacity (Section 3.2) to assess its contribution. Beamforming gain is also expressed in terms of the spacing between transmitting antennas (Section 3.3) and the MIMO channel correlation coefficient (Section 3.4).

\subsection{RECEIVED POWER}

The received power is an important parameter determining the transmission quality. In fact, the bit error rate (BER) decreases as the received power increases, which helps maintaining a high data rate. Otherwise, the degradation of the bit error rate causes a switching to a modulation and coding scheme that reduces the bit rate. Enabling the beamforming option can compensate a possible decrease of the received power due to a deterioration of transmission conditions.

The powers received by all the $\mathrm{Rx}$ antennas in the cases of activation and no activation of TxBF are compared. The transmission scheme with a 4 transmitting antennas system is equivalent to the system shown in Figure 3.

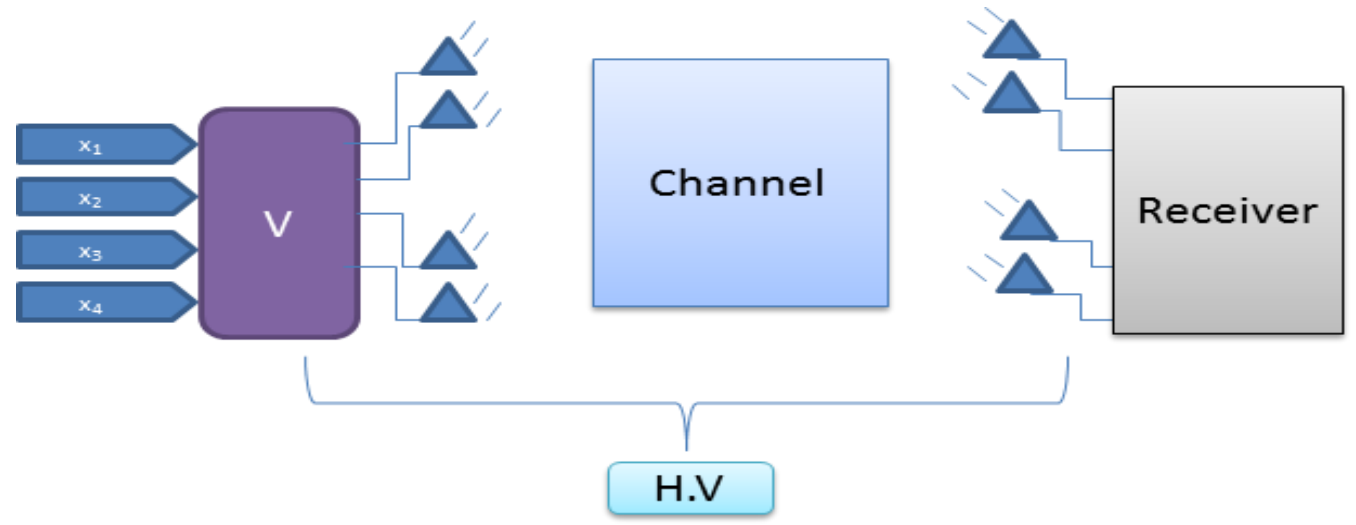

Figure 3. Transmission scheme in case of an SVD precoding.

It is obvious that the channel matrix $H$ is replaced by a matrix $H V$.

We consider a simple TxBF scheme that does not use water filling to adapt the power of the transmitted signals. So, each independent spatial stream has the same average power $\mathrm{E}\left[\left|x_{i}\right|^{2}\right]=\frac{P_{e}}{N_{s s}}$, where $\mathrm{E}[$.$] is the statistical mathematical expectation operator, P_{e}$ is the total emitted power on each OFDM carrier and $N_{s s}$ is the number of spatial streams. For our numerical examples, $P_{e}=20 \mathrm{~mW}$ over $2 \mathrm{MHz}$ bandwidth, which is a reasonable value for $802.11 \mathrm{ac} / \mathrm{n}$ devices in the $5 \mathrm{GHz}$ frequency band (typically $200 \mathrm{~mW}$ over $20 \mathrm{MHz}$ ). Therefore, using (4), the expression of the instantaneous received power $P_{W-B F}$ by the Rx antenna array becomes

$$
P_{W-B F}=\frac{P_{e}}{N_{s s}} \operatorname{Trace}\left(H V V^{*} H^{*}\right)=\frac{P_{e}}{N_{s S}} \sum_{k=1}^{N_{S S}}\left|s_{k}\right|^{2}
$$

For all the results presented in this paper, the same total transmitted power $P_{e}$ is used, whatever are the values of $N_{s s}, N T x$ or $N R x$. MIMO configuration is defined later in this paper by the triplet $N_{s s} \times N R x \times N T x$. 
In the case of a link without $\mathrm{TxBF}$, the transmission scheme is given Figure 4 . For this scheme, $Q$ is the mapping matrix used when there are more transmit antennas than spatial streams $\left(N_{S S}<N T x\right)$, as in [18].

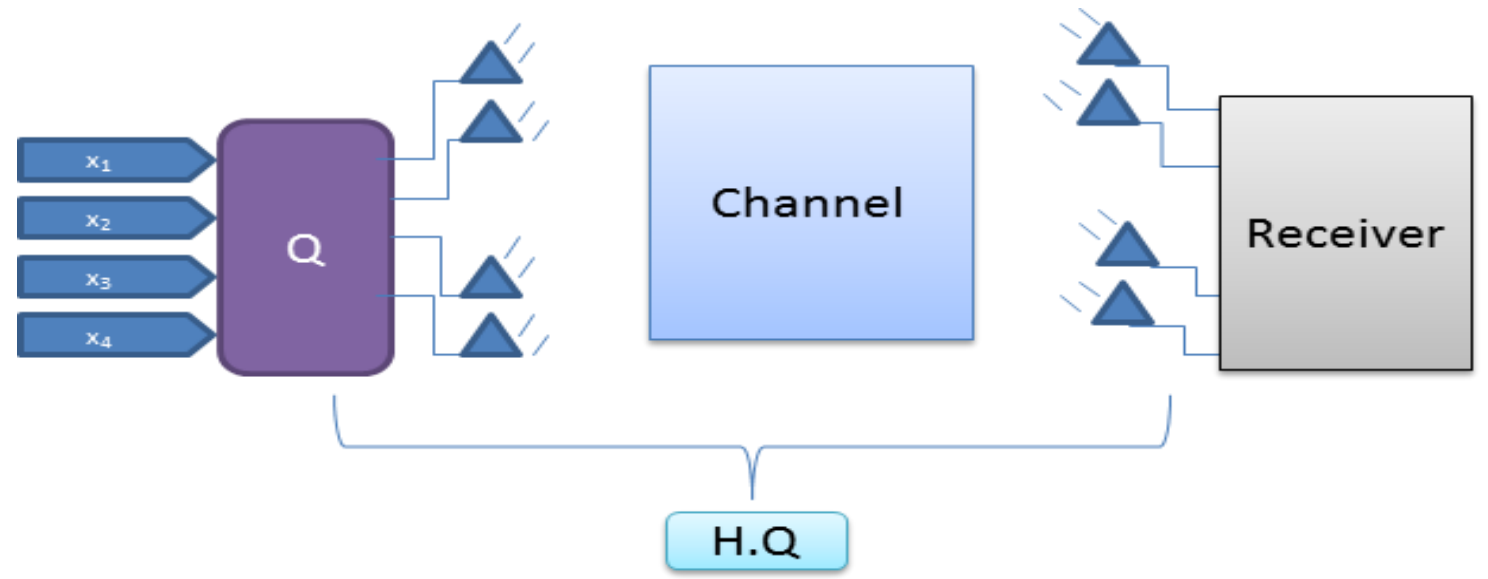

Figure 4. Transmission scheme without BF.

For example, for a $4 \times 4$ MIMO system with 2 spatial streams

$$
Q=\frac{1}{\sqrt{2}}\left(\begin{array}{ll}
1 & 0 \\
0 & 1 \\
1 & 0 \\
0 & 1
\end{array}\right)
$$

Using (1), the received power without BF is

$$
P_{W O-B F}=\frac{P_{e}}{N_{s s}} \operatorname{Trace}\left(H Q Q^{*} H^{*}\right)
$$

With a simple calculation using (5) and (7), it can be shown that if the number of spatial streams is equal to the number of transmitting antennas, then the received power with TxBF is identical to that received without BF, i.e. if $N_{S S}=N T x$, then $P_{W O-B F}=P_{W-B F}$. Therefore, later in the paper, the relevant cases to be considered when studying the received power correspond to $N_{S S}<N T x$.

In a first step, we have studied a $4 \times 4$ MIMO system. The aim is to compare the received power when we consider 1, 2 and 3 spatial streams with and without BF.

In a second step, we fix the number of spatial streams $N_{S S}=2$ and then we compare different MIMO configurations in terms of received power. Results are given using 250 realizations of the channel matrix $H$. The complementary cumulative distribution functions (CCDF) are displayed in Figures 5 and 6.

By using BF we expect some gains concerning the median or average received power thanks to the Tx antenna array gain and also a reduced fading depth (or improved diversity gain) thanks to an optimized recombination of the multipaths. Consequently, the results drawn in Figure 5 are analyzed by considering the BF gain (received power difference with and without BF) for two probability levels 0.5 and 0.9 . 


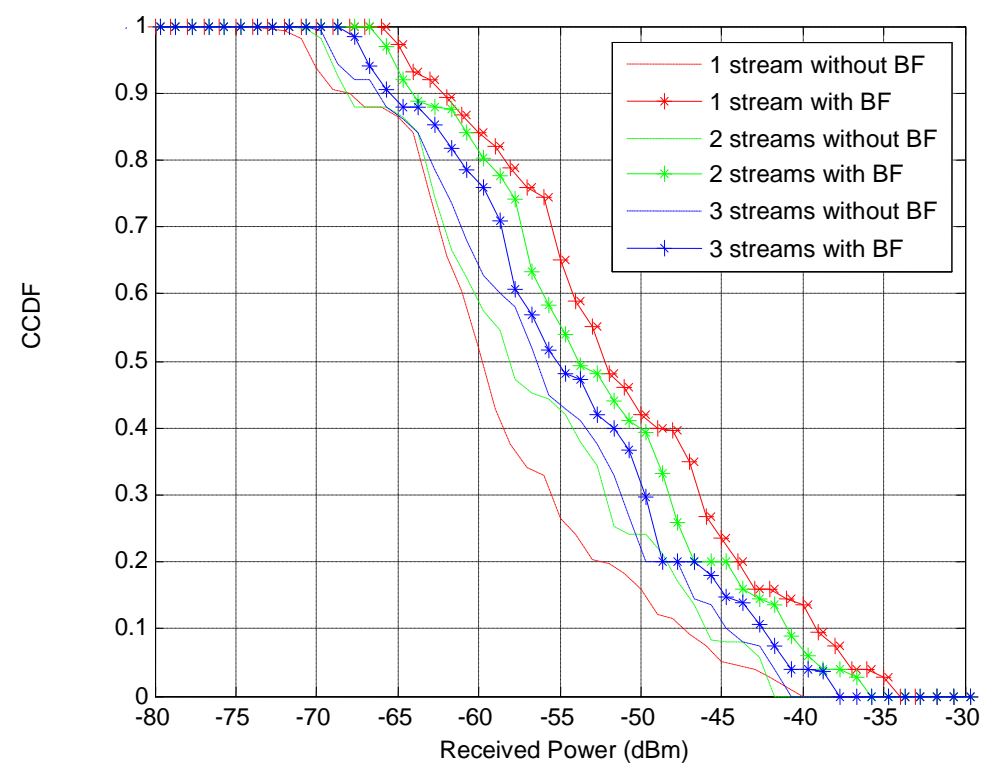

Figure 5. Comparison of CCDF of the received power for 1, 2 and 3 spatial streams on $4 \times 4$ MIMO system.

- For the 0.5 probability value, the median difference between the received power with $\mathrm{BF}(\mathrm{W}-\mathrm{BF})$ and without $\mathrm{BF}$ (WO-BF) is computed. The median received power gain becomes smaller when the number of spatial streams increases. It remains, nevertheless, more important than the gain computed for a MIMO uncorrelated Rayleigh channel (Figure 7). This is probably because the simulated channel is more correlated and with less scattering than for Rayleigh channel.

- The fading depth reduction is computed as the difference between the received powers $\mathrm{W}-\mathrm{BF}$ and $\mathrm{WO}-\mathrm{BF}$ for the 0.9 threshold probability. It becomes smaller when the number of spatial streams increases. The diversity gain for a Rayleigh channel (Figure 7) is more important than with our simulated channel, probably because of the same reasons as previously. A similar trend was observed in the case of mm-wave in the $60 \mathrm{GHz}$ frequency band [19].

Based on analysis of the received powers computed when $N_{s s}=2$, Figure 6 shows that the $\mathrm{BF}$ improves the received power in most of the studied configurations. This improvement is more sensitive to the number of receiving antennas because the configurations $4 \times 4$ and $3 \times 4$ show better performance compared to $4 \times 3$ and $2 \times 4$ configurations. 


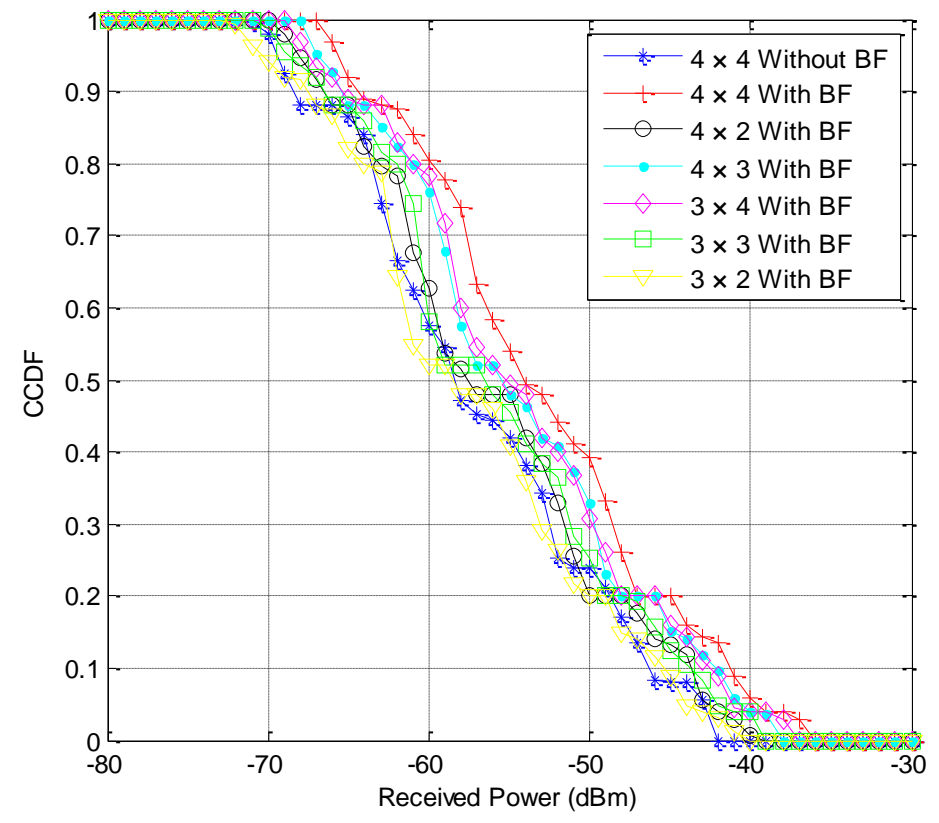

Figure 6. Comparison of CCDF of the received power for several MIMO configurations when $N_{S S}=2$.

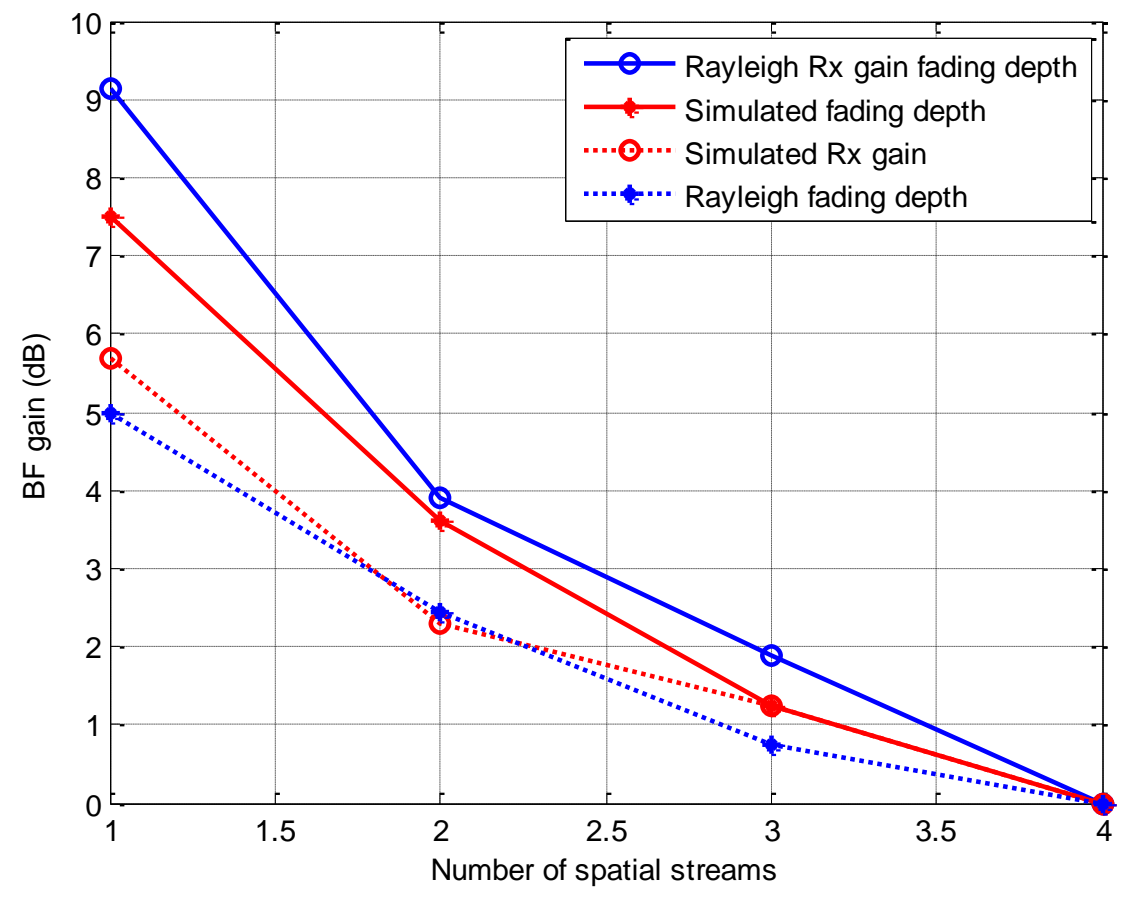

Figure 7. Median received power gain and fading depth reduction for simulated Rayleigh and indoor residential channel. 


\subsection{SNR AND CHANNEL CAPACITY}

In this section, the $S N R$ each spatial stream is analyzed in order to emphasize the effect of $\mathrm{BF}$ activation. In the case of multiple streams, the channel capacity is computed and compared with the case of a simple MIMO system.

The transmission chain in the W-BF case is presented by Figure 8 , where $X$ is the transmitted signal, $\sigma_{n}$ is the standard deviation (RMS value) of the additive Gaussian noise $n$, while $V$ is the precoding matrix used for TxBF deduced from SVD applied to $H$ and after selection of $N_{s s}$ spatial streams. $\mathrm{ZF}$ is the zero forcing receiver.

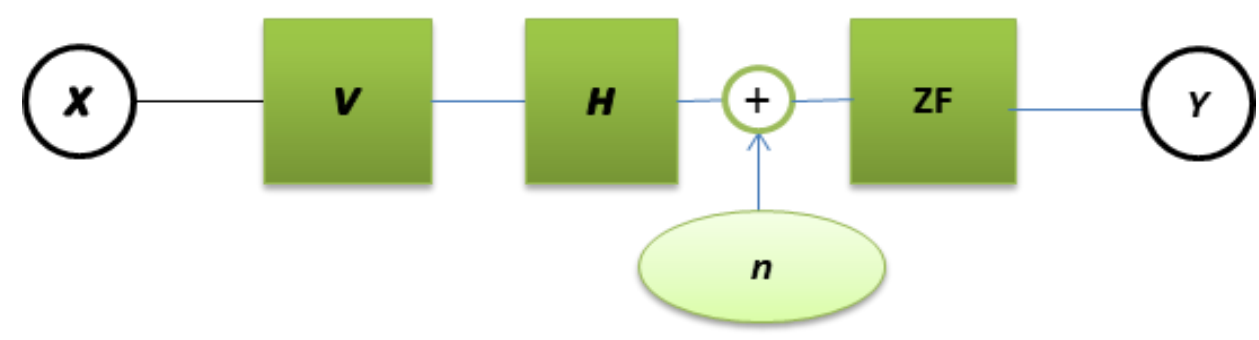

Figure 8. Transmission scheme with MIMO-SVD.

Given the nature of the studied environment, the Tx-Rx distances are small, the signal attenuation is limited and eventually the $S N R$ s are high, which justifies the use of ZF since it is equivalent to the Minimum Mean Square Error (MMSE) equalizer at high SNR. It is also a case in favor of using the beamforming. It has been shown in [20] that a $\mathrm{ZF}$ receiver is equivalent in terms of $S N R$ to a receiver that applies the $U^{*}$ matrix associated to the SVD of H.

The estimated signal $X_{\text {est }}$ (after detection) is

$$
X_{\text {est }}=X+\left((H V)^{*} H V\right)^{-1}(H V) n
$$

with $\left((H V)^{*} H V\right)^{-1}=\left(S^{*} S\right)^{-1}$.

By applying the $U^{*}$ matrix at the reveiver, the estimated signal would be $X_{\text {est }}=S X+U^{*} n$, which leads exactly to the same $S N R$ as the ZF receiver.

The noise covariance matrix $\operatorname{cov}(n)$ at the ZF output becomes $\operatorname{cov}(n)=\sigma_{n}^{2}\left((H V)^{*} H V\right)^{-1}$.

With TxBF, the $S N R_{W-B F, i}$ of each stream can be written as

$$
S N R_{W-B F, i}=s_{i}^{2} \frac{E\left[\left|x_{i}\right|^{2}\right]}{\sigma_{n}^{2}}
$$

where $s_{i}$ is the $i$-th singular value.

By analogy with the foregoing, in the case without $\mathrm{TxBF}$, the matrix $Q$ replaces $V$ and the $S N R$ of each stream becomes

$$
S N R_{W O-B F, i}=\frac{E\left[\left|x_{i}\right|^{2}\right]}{\sigma_{n}^{2} \cdot \operatorname{Diag}_{i}\left(\left((H Q)^{*}(H Q)\right)^{-1}\right)}
$$

The same statistical analysis as in the previous paragraph has been made using the 250 MIMO channel realizations. The obtained results are given in Figure 9 and Figure 10. 
In order to compute $S N R$, we consider for numerical examples a noise temperature $\mathrm{T}=$ $300{ }^{\circ} \mathrm{K}$ and a noise spectral density of $-173.8 \mathrm{dBm} / \mathrm{Hz}$, so that $\sigma_{\mathrm{n}}{ }^{2}=-110.8 \mathrm{dBm}$ for a $2 \mathrm{MHz}$ frequency band.

For the $4 \times 4$ MIMO system, the results given in Figure 9 show that BF activation improves the $S N R$ of the three main spatial streams with a $S N R$ degradation of the fourth stream.

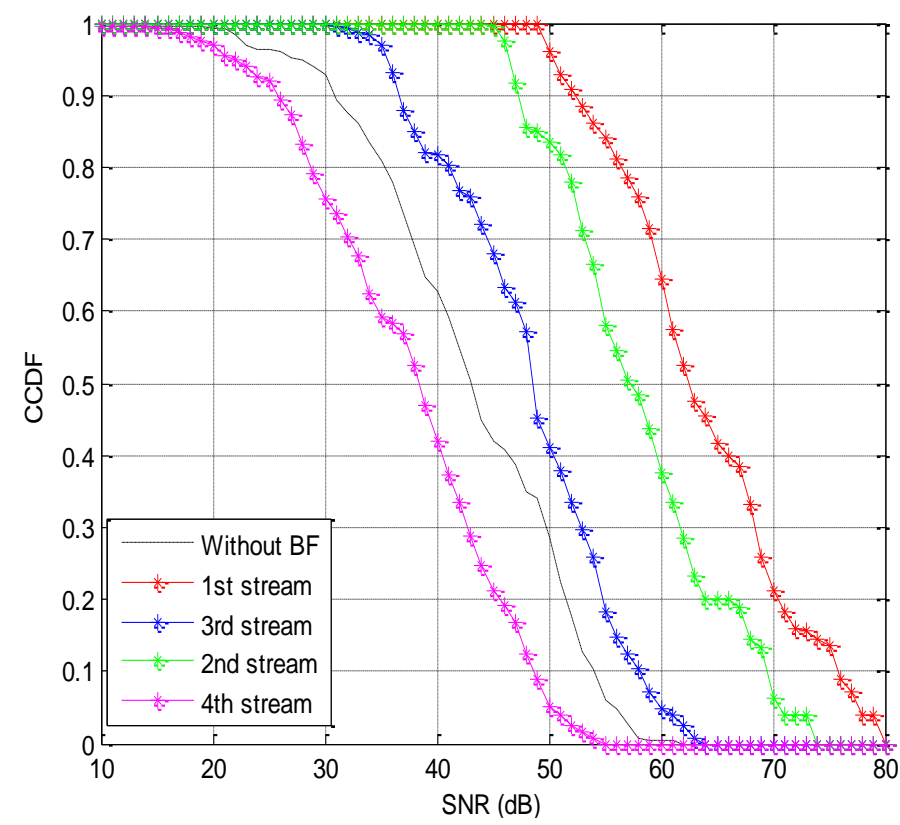

Figure 9. Comparison of CCDF of SNR for each spatial stream in a $4 \times 4$ MIMO configuration with 4 spatial streams.

To compare the different MIMO configurations, the channel capacity was calculated using (11) from [21]

$$
C=\sum_{k=1}^{N_{S S}} \log _{2}\left(1+S N R_{k}\right) \quad(\mathrm{bit} / \mathrm{s} / \mathrm{Hz})
$$

where $S N R_{k}$ is the signal to noise ratio of the $k^{\text {th }}$ spatial stream.

The comparison of different MIMO configurations presented in Figure 10 shows that the TxBF activation improves the channel capacity in almost all the studied configurations, except for the $2 \times 4 \times 2$ ( 2 spatial streams on a $4 \times 2 \mathrm{MIMO}$ ). For a given $N_{s s}$, we also find that an increase in the number of receiving antennas is more advantageous for the channel capacity compared to the number of transmitting antennas. 


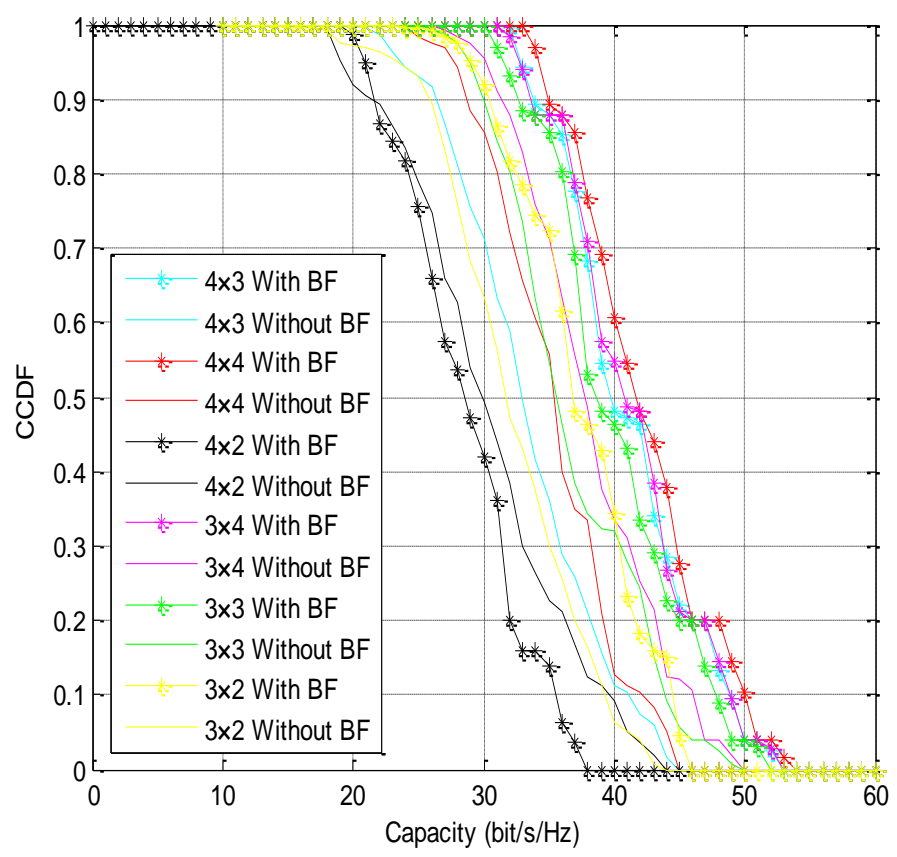

Figure 10. Comparison of CCDF of channel capacity for several MIMO configuration when $N_{S S}=2$.

It remains an issue to be addressed; for a given MIMO configuration, is it better to increase the number of transmit /receive antennas or the number of spatial streams and is there enough multipath diversity to use more spatial streams? To answer this question, the same comparative study as for $N_{s s}=2$ was performed for $N_{s s}=3$ (Figure 11). The results show that increasing the number of spatial streams has a greater effect on the channel capacity compared to a simple increase of the number of transmitting and receiving antennas. For example, the average capacity for a $2 \times 3 \times 3 \mathrm{MIMO}$ configuration is $38 \mathrm{bit} / \mathrm{s} / \mathrm{Hz}$ with $N_{s s}=2$. Capacity goes to $52 \mathrm{bit} / \mathrm{s} / \mathrm{Hz}$ when $N_{s s}=3$ but it is only $42 \mathrm{bit} / \mathrm{s} / \mathrm{Hz}$ for a $2 \times 4 \times 4$ configuration.

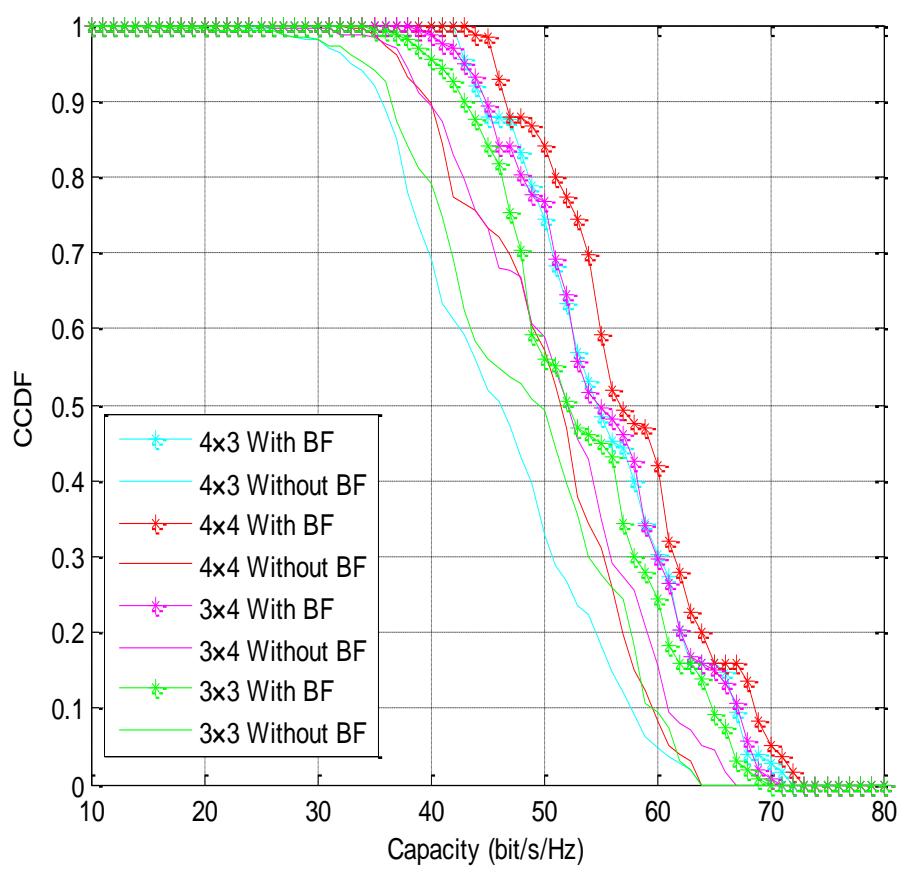

Figure 11. Comparison of CCDF of channel capacity for several MIMO configuration when $N_{S S}=3$. 


\subsection{BEAMFORMING GAIN (BFG)}

Beamforming gain is defined as the average difference between the received power (expressed in $\mathrm{dB}$ ) with TxBF and without TxBF for the same $N_{s s}, N T x$ and NRx MIMO configuration

$$
B F G=10 \log _{10}\left(\frac{P_{W-B F}}{P_{W O-B F}}\right)
$$

$B F G$ is evaluated in terms of the spacing between transmitting antennas for different MIMO configurations (different numbers of $\mathrm{Tx}$ and $\mathrm{Rx}$ antennas). The number of spatial streams is fixed: $N_{s s}=2$. This is the basic value required for $802.11 \mathrm{n}$ access points, higher values are optional. The objective is to identify which of these configurations achieves the maximum gain when the Tx antenna spacing changes.

For a first position of the transmitter (in the corridor), six $d T x$ (transmit antenna spacing) values have been considered for the simulations: $0.25 \lambda, 0.5 \lambda, 0.75 \lambda, \lambda, 1.25 \lambda$ and $1.5 \lambda$, where $\lambda$ is the carrier wavelength. The gain is given by Figure 12. Two remarks may be relevant for this example: the first concerns the fact that the maximum $B F G$ is achieved for two different spacings between the transmitting antennas: near $0.5 \lambda$ and near $1.25 \lambda$. The second is that the maximum gain is not necessarily achieved when using a large number of transmitting and receiving antennas; the configurations $2 \times 4$ and $4 \times 3$ are more suitable to achieve a significant gain by activating TxBF than $4 \times 4$.

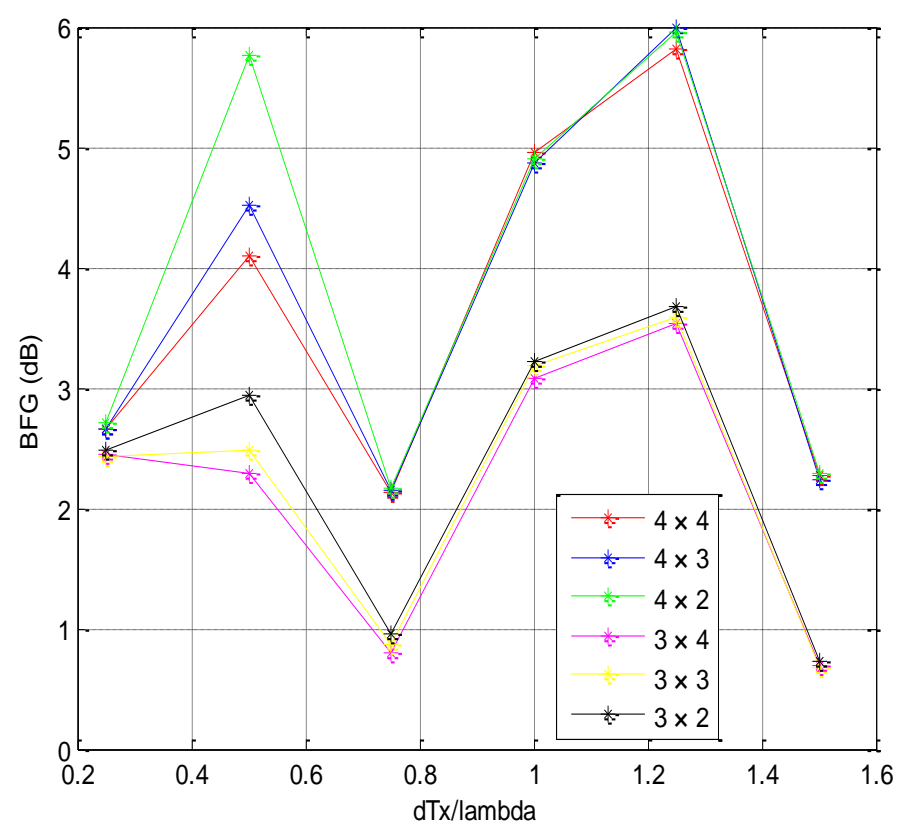

Figure 12. BFG versus transmit antenna spacing for several MIMO configurations. (Transmitter in the corridor)

To confirm the results observed in the case of a transmitter located in the corridor, other simulations have been performed for a transmitter located in the living room (Figure 13). Almost the same trends are obtained. To generalize this result to other environments, further simulations or measurements would be useful in future works. 


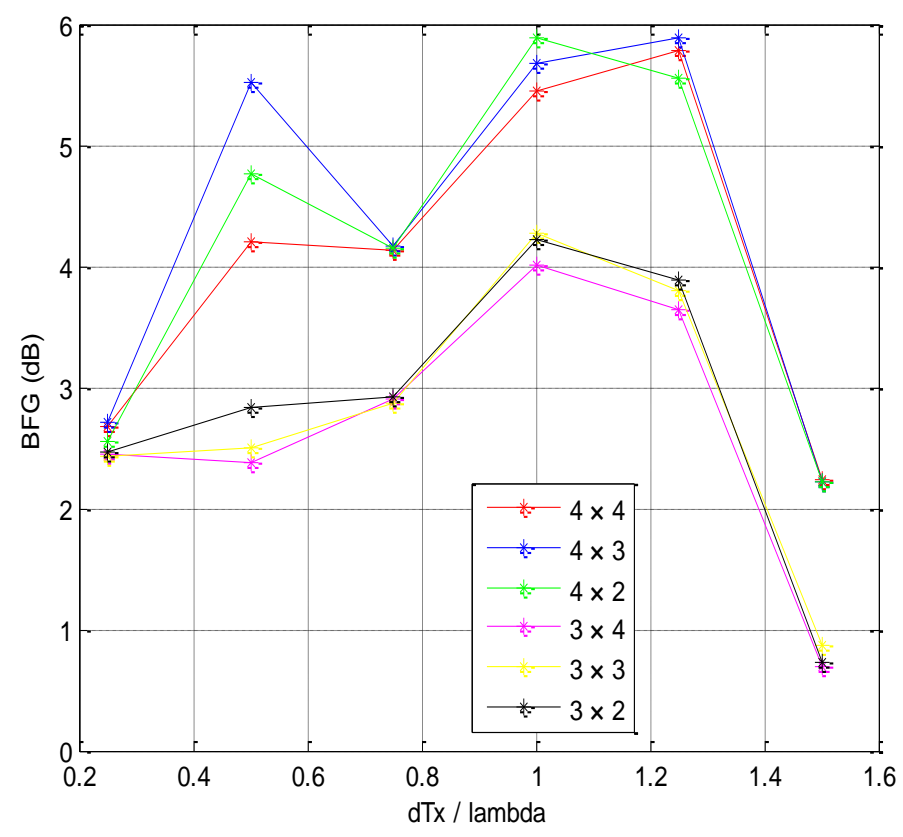

Figure 13. BFG versus transmit antenna spacing for several MIMO configurations. (Transmitter in the living room)

From these results, it can be observed that the activation of TxBF when $N_{S S}=2$ is important if NTx is great and NRx is small. In the opposite case, the TxBF does not have a great interest.

For practical deployment, one of the performance criteria to take into account is the coverage area of the system. These results can be interpreted in terms of improvement of it, as the activation of beamforming improves the received power. In fact, in [22] the typical propagation path loss (in $\mathrm{dB}$ ) can be expressed as follows

$$
P L=A_{0}+B \log _{10}\left(\frac{d}{d_{0}}\right)
$$

where $A_{0}$ and $B$ are two constants, $\mathrm{d}$ is the distance between transmitter and receiver and $d_{0}$ is a reference distance $\left(d_{0}=1 \mathrm{~m}\right)$. The antenna gains are taken into account in the constants values. The received power (in $\mathrm{dBm}$ ) in case of $\mathrm{BF}$ can be written as follows

$$
P_{W-B F}=P e-A_{0}-B \log _{10}\left(\frac{d}{d_{0}}\right)+B F G
$$

where $B F G$ is the beamforming gain. In a basic MIMO case, the power is

$$
P_{W O-B F}=P e-A_{0}-B \log _{10}\left(\frac{d}{d_{0}}\right)
$$

We search the maximum transmission ranges for a same reception level threshold $S_{\min }$ to show how TxBF can improve the coverage area of MIMO systems. $d_{W-B F}$ and $d_{W O-B F}$ are these maximum transmission ranges $\mathrm{W}-\mathrm{BF}$ and $\mathrm{WO}-\mathrm{BF}$ respectively to reach $P_{W O-B F}=$ $P_{W-B F}=S_{\min }$. From (14) and (15), it can be deduced that

$$
\frac{d_{W-B F}}{d_{W O-B F}}=10^{\frac{B F G}{B}}
$$


In typical indoor environments, the path loss models consider a value of $B$ either 20 or 35 depending on LOS or NLOS, such as in the TGN channel model [23].

When $B=35$, with $4 \times 2$ MIMO configuration, the coverage range gain is about $10^{\frac{5.7}{35}}=1.45$. For instance, the distance between the transmitter and the receiver can go from $10 \mathrm{~m}$ to $14.5 \mathrm{~m}$ for the same received power in NLOS conditions, which results in a transmission range gain of more than $45 \%$.

When $B=20$, then $10^{\frac{5.7}{20}}=1.92$ and the transmission range gain reaches $92 \%$.

Table 1 gives the transmission range gain for the studied MIMO configurations using the results of Figure 12.

TABLE 1

TRANSMISSION RANGE GAIN

\begin{tabular}{lcc}
\hline \hline MIMO & BFG $($ LOS, B = 20) & BFG (NLOS, B = 35) \\
\hline $4 \times 4$ & $60.32 \%$ & $30.96 \%$ \\
$4 \times 3$ & $67.88 \%$ & $34.45 \%$ \\
$4 \times 2$ & $92.75 \%$ & $45.50 \%$ \\
$3 \times 4$ & $30.32 \%$ & $16.34 \%$ \\
$3 \times 3$ & $32.59 \%$ & $17.49 \%$ \\
$3 \times 2$ & $39.64 \%$ & $21.02 \%$
\end{tabular}

\subsection{BFG AND CHANNEL CORRELATION}

The MIMO channel correlation matrices $R T x$ (at the transmit side) and $R R x$ (at the receive side) are calculated using a centered and normalized matrix $H_{\text {norm }}$ deduced from the channel matrix $\mathrm{H}$ based on the following formulas [24]

$$
\begin{aligned}
& R R x=\frac{1}{N T x} \cdot \frac{1}{N} \sum_{k=1}^{N} H_{\text {norm }}(k) H_{\text {norm }}(k)^{*} \\
& R T x=\frac{1}{N R x} \cdot \frac{1}{N} \sum_{k=1}^{N} H_{\text {norm }}(k)^{T} \operatorname{conj}\left(H_{\text {norm }}(k)\right)
\end{aligned}
$$

where $N=10$ is the number of channel samples (number of OFDM carriers in this paper). $H_{\text {norm }}(k)^{T}$ is the transposed form of $H_{\text {norm }}(k),(.)^{*}$ is the Hermitian form. $H_{\text {norm }}(k)$ is the $k^{\text {th }}$ frequency realization of the centered and normalized channel matrix $H_{n o r m}$ and conj(.) is the conjugate operator.

The centered and normalized coefficient $\mathrm{h}_{\text {norm }}^{\mathrm{ij}}(\mathrm{k})\left(\mathrm{i}^{\text {th }}\right.$ row and $\mathrm{j}^{\text {th }}$ column) of the $H_{\text {norm }}(k)$ matrix is computed using

$$
\mathrm{h}_{\text {norm }}^{\mathrm{ij}}=\frac{\mathrm{h}^{\mathrm{ij}}(\mathrm{k})-\mathrm{m}^{\mathrm{ij}}}{\sigma^{\mathrm{ij}}}
$$

where

$$
\mathrm{m}^{\mathrm{ij}}=\frac{1}{N} \sum_{k=1}^{N} \mathrm{~h}^{\mathrm{ij}}(\mathrm{k})
$$


and

$$
\sigma^{\mathrm{ij}}=\sqrt{\frac{1}{N} \sum_{k=1}^{N}\left|\mathrm{~h}^{\mathrm{ij}}(\mathrm{k})-\mathrm{m}^{\mathrm{ij}}\right|^{2}}
$$

A single correlation coefficient CorTx is calculated using $R R x$ and $R T x$ by averaging the non-diagonal coefficients. This CorTx coefficient is deduced from $R T x$. For example, in the case of a $4 \times 4$ MIMO system and when

$$
R T x=\left(\begin{array}{cccc}
1 & \rho_{12} & \rho_{13} & \rho_{14} \\
\rho_{21} & 1 & \rho_{23} & \rho_{24} \\
\rho_{31} & \rho_{32} & 1 & \rho_{34} \\
\rho_{41} & \rho_{42} & \rho_{43} & 1
\end{array}\right)
$$

The average coefficient CorTx is given by

$$
\operatorname{CorTx}=\frac{\left|\rho_{12}\right|+\left|\rho_{13}\right|+\left|\rho_{14}\right|+\left|\rho_{23}\right|+\left|\rho_{24}\right|+\left|\rho_{34}\right|}{6}
$$

Some previous studies [25] have shown that the dominant mode eigen beamforming has the highest gain when channel average correlation coefficient is high (single spatial stream), but no results are available concerning the more general case SVD-MIMO with multiple spatial streams. In the following, we give an example of results to analyze the relation between BFG and CorTx for $d T x=1.25 \lambda$, in the case of single or multiple spatial streams. For this value of $d T x$, it has been shown previously that a maximum BFG value can be reached.

Up to 3 independent spatial streams on a $4 \times 4$ MIMO configuration are considered in this simulation. BFG is assessed for various $N_{s s}$ in terms of the average correlation coefficient CorTx.

For the cases with 1 or 2 spatial streams over $4 \times 4$ MIMO channel, the beamforming gain increases when the correlation coefficient increases. In other words, when the environment becomes increasingly correlated (near like LOS correlation conditions), BFG tends to increase. In the case of $N_{s s}=3$ with a $4 \times 4$ MIMO system, slightly the same tendency can be observed but more $N_{s s}$ is high and less is BFG correlated with CorTx (Figure 14).

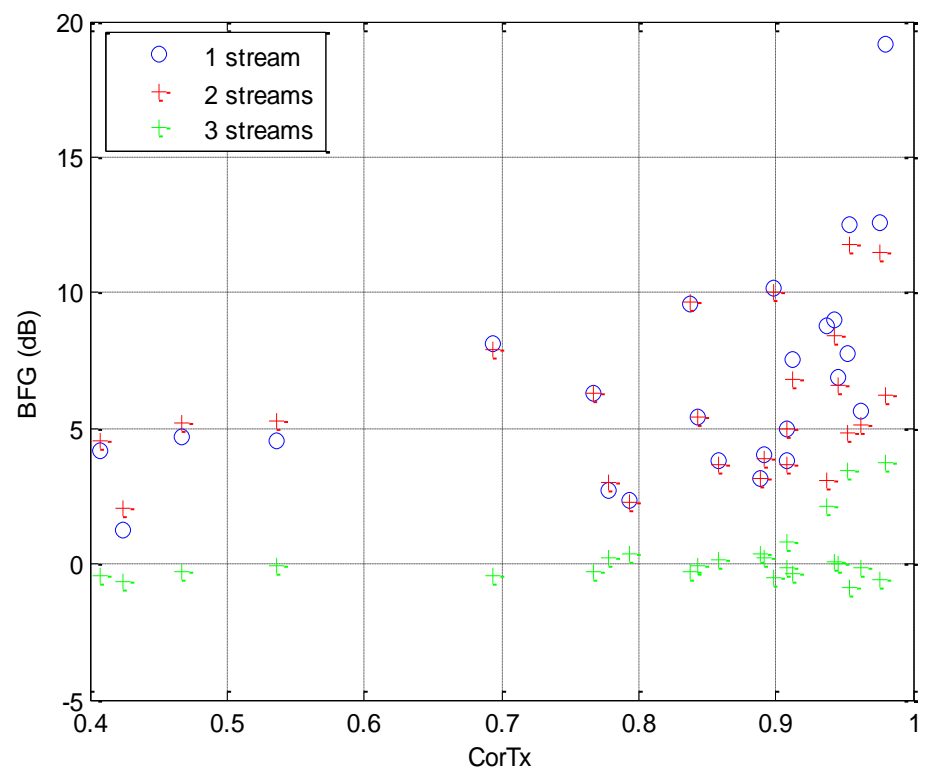

Figure 14. BFG versus CorTx for 1, 2, and 3 streams on a $4 \times 4$ MIMO system. 


\section{Beamforming and Interference}

One other relevant aspect to be addressed about transmit beamforming is its ability to reduce interference. It modifies the radiation of the emission and favors the targeted receiver, but it is unclear if the power level of other receivers has increased, as this depends on the multipath, the number of antennas and the beam directivity. The considered TxBF optimizes the $S N R$ but is not designed to maximize the SIR. Indeed, the purpose of this part is to investigate whether the introduction of transmit BF can increase or reduce the effect of interference generated by a jamming link. Several cases can be considered; the main link and the interfering link can both use or not the transmit beamforming option. The TxBF option is set to "active" for the interfering links. Therefore we compare the SIR with and without activation of the BF on the main link. This study was performed using a standard receiver without optimization, i.e. without taking into account the interference reduction.

The considered transmission scheme is given in Figure 15. In this figure, the link between $X_{2}$ data and $Y_{2}$ is the main link (link 2), while the interfering link is between $X_{1}$ and $Y_{1}$ (link 1). If both wireless links (transmitter, receiver) use the SVD pre-coding technique, two $V$ precoding matrices are introduced, one for each transmitter.

The MIMO channel matrix between the transmitter $X_{1}$ and the receiver $Y_{2}$ is denoted $H_{12}$. $n_{1}$ and $n_{2}$ are independent additive complex Gaussian noises of zero mean with the same variance.

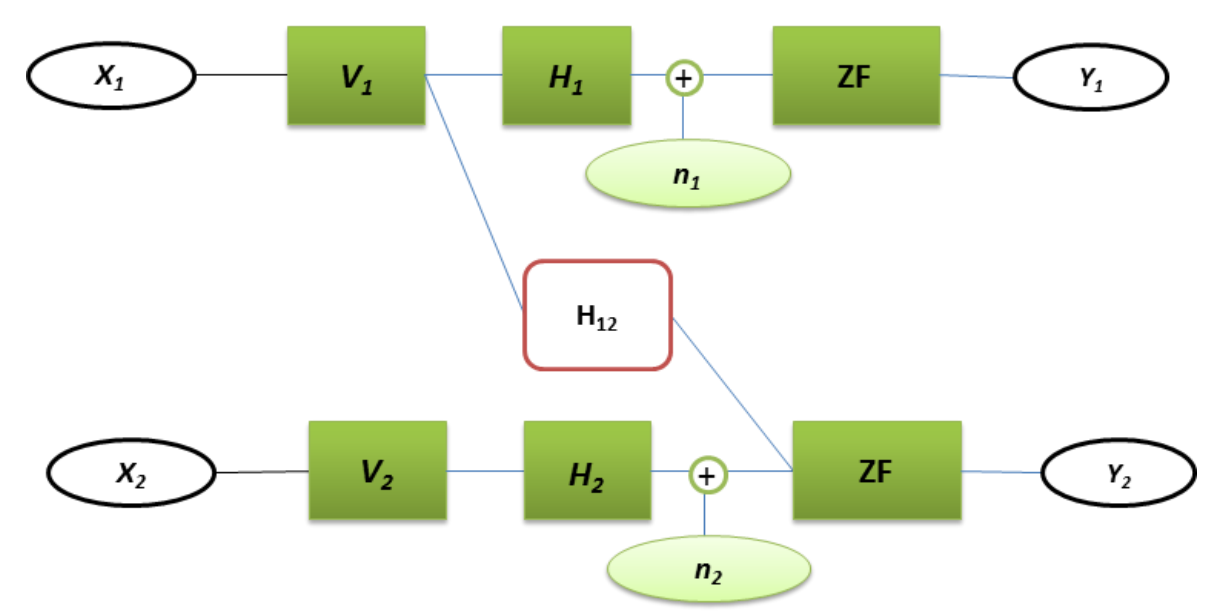

Figure 15. Transmission scheme with an interfering link and beamforming.

Both links are using the same frequency channel (co-channel links). In the case of adjacent channels, an additional constant rejection coefficient due to filtering would have to be considered and applied to the interference power, but the conclusions would not be modified. term is

At the output of a ZF equalizer (zero forcing), the general expression of the interference

- with BF:

$$
J_{W_{-} B F}=\left[\left(H_{2} V_{2}\right)^{*}\left(H_{2} V_{2}\right)\right]^{-1}\left(H_{2} V_{2}\right)^{*}\left(H_{12} V_{1}\right) X_{1}
$$


- and without BF:

$$
J_{W O_{-} B F}=\left[\left(H_{2} Q_{2}\right)^{*}\left(H_{2} Q_{2}\right)\right]^{-1}\left(H_{2} Q_{2}\right)^{*}\left(H_{12} V_{1}\right) X_{1}
$$

where $X_{1}$ is the transmitted vector and $Q_{2}$ is the mapping matrix of the link 2 . As we have

$$
H_{2} V_{2}=U_{2} S_{2} V_{2}^{*} V_{2}=U_{2} S_{2}
$$

And

$$
V_{2}^{*} V_{2}=I_{N T x \times N T x}
$$

so

$$
\left(H_{2} V_{2}\right)^{*} H_{2} V_{2}=S_{2}^{*} U_{2}^{*} U_{2} S_{2}=S_{2}^{*} S_{2}
$$

and finally

$$
J_{W_{-} B F}=\left[S_{2}\right]^{-1}\left(U_{2}{ }^{*} H_{12} V_{1}\right) X_{1}
$$

The total interferences power is then

$$
\begin{gathered}
P_{J}^{W-B F}=\operatorname{Trace}\left(R_{J}^{W-B F}\right) \\
P_{J}^{W O-B F}=\operatorname{Trace}\left(R_{J}^{W O-B F}\right)
\end{gathered}
$$

where

$$
R_{J}^{W-B F}=\mathrm{E}\left[J_{W_{-} B F} J_{W_{-} B F^{*}}{ }^{W}\right.
$$

and

$$
R_{J}^{W O-B F}=\mathrm{E}\left[J_{W O_{-} B F} J_{W O_{-} B F}{ }^{*}\right]
$$

To compare the interference power level and the main link signal power, the $S I R_{i}$ (signal to interference ratio) is calculated for each spatial stream

$$
\begin{gathered}
S I R_{i}{ }^{W-B F}=\frac{P_{S}}{R_{J}^{W-B F}(i, i)} \\
S I R_{i}{ }^{W O-B F}=\frac{P_{S}}{R_{J}^{W O-B F}(i, i)}
\end{gathered}
$$

where

$$
P_{s}=\frac{P_{e}}{N_{s s}}
$$

SIR was computed for a $4 \times 4$ MIMO configuration with $N_{s s}=3$ for both links. The SIR of each stream is calculated and compared to the corresponding value obtained without the $\mathrm{BF}$ 
use. The complementary cumulative distribution function is then plotted (Figure 16 and Figure 17). The same previously described environment has been used. The link between one transmitter (Transmit2 in Figure 1) and one of the 25 receivers (rec25) for LOS and rec3 for NLOS has been chosen as the main link. The links between the second transmitter (Transmit1) and the remaining 24 receivers were seen as interfering links. The channel matrices are calculated for 10 subcarriers such as previously stated. Thus statistics were performed for 240 (10 subcarriers $\times 24$ interfering configurations) realizations of the matrix $H$ of the main link.

Figure 16 and Figure 17 compare the CCDF of SIR with and without beamforming in the case of a main link in NLOS (Figure 16) and LOS (Figure 17). In 90\% of the realizations of the first spatial stream, SIR is improved by $9 \mathrm{~dB}$ in NLOS conditions. The same remarks can be drawn in the case of LOS conditions except that SIR is significantly higher as the desired $\mathrm{Rx}$ is closer to its transmitter.

The difference between $S I R$ with and without TxBF is tightening for the second or the third spatial streams. Thus TxBF improves SIR especially when the main link corresponds to LOS conditions.

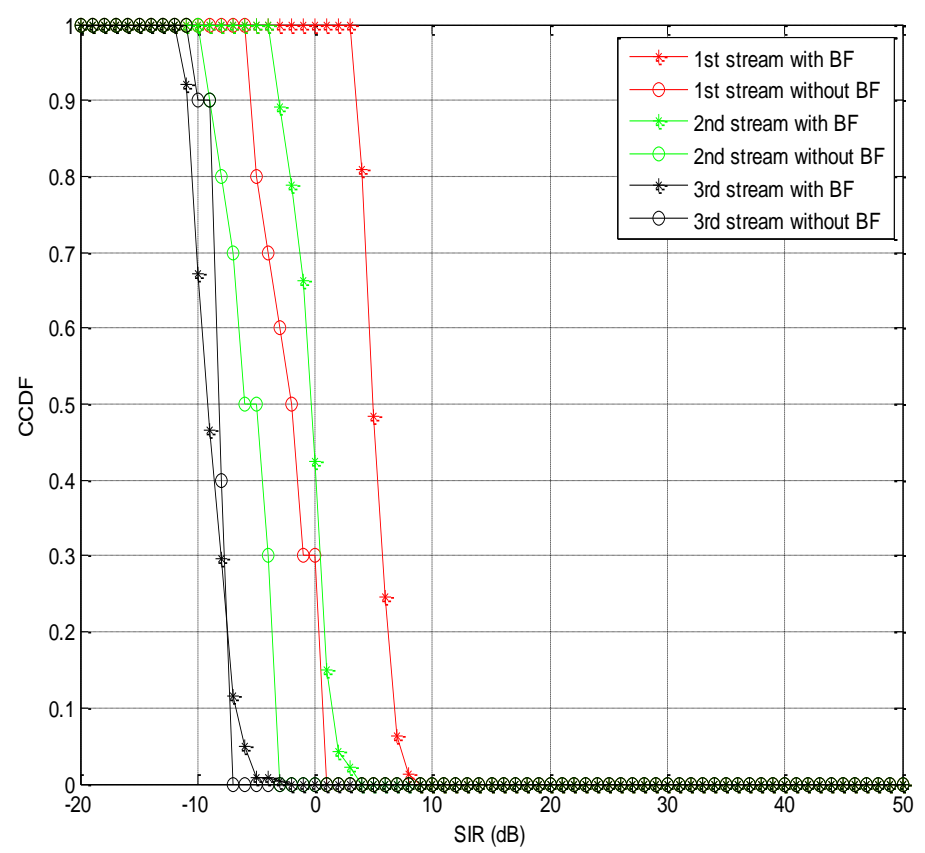

Figure 16. CCDF of SIR with NLOS condition in main link, 3 streams on $4 \times 4$ MIMO system. 


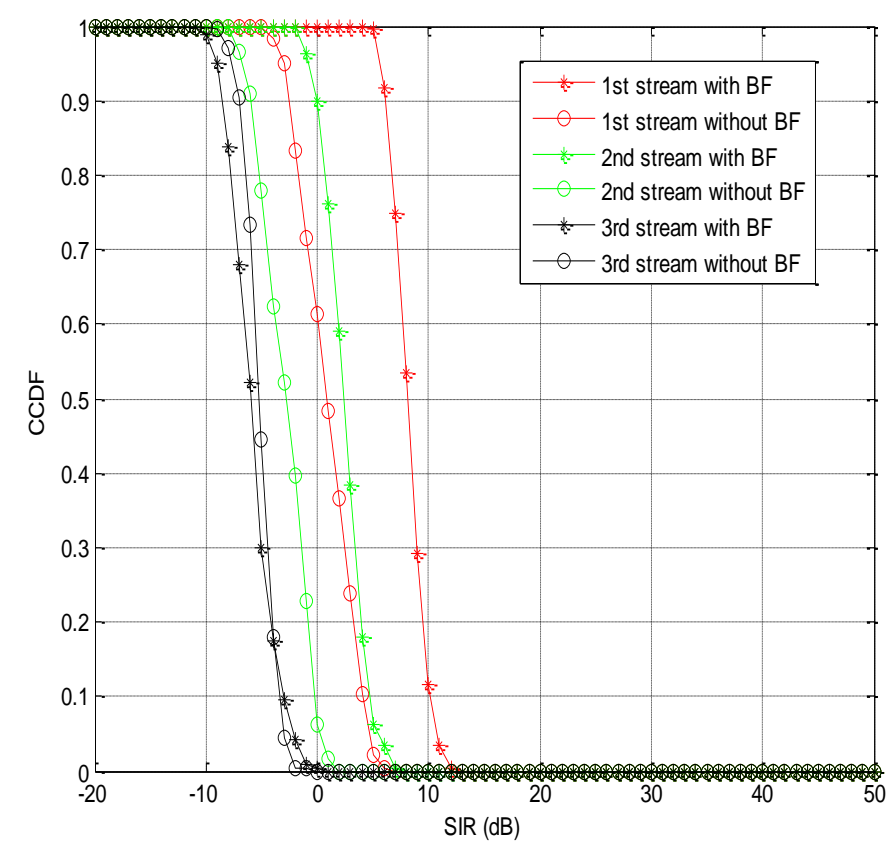

Figure 17. CCDF of SIR with LOS condition in the main link, 3 spatial streamson $4 \times 4$ MIMO system.

\section{Conclusion}

For indoor environments, MIMO systems are the basis of the increased data rate, at least theoretically. In practice, the multipath propagation channel is behind the performance degradation. However, the use of several options provided by system standards such as IEEE802.11n/ac may bring several solutions. Therefore, the transmit beamforming is analyzed in this paper. Simulations were performed for a typical residential environment. The matrices of the propagation channels were calculated using a ray tracing tool. The SVD precoding algorithm has been added to the transmission chain. The obtained results show that enabling TxBF increases the received power when $N T x$ is important compared to $N_{s s}$ (if $N_{s s}=N T x$ there is no power gain). The received power gain is greater than that of a Rayleigh channel, but the fading depth reduction is less important because the channel corresponding to the studied environment is probably less scattered. Concerning $S N R$, for a given $N_{s s}$, it was shown that with TxBF enabled, it is more advantageous to increase the number of $\mathrm{Rx}$ antennas than the Tx antennas number. The residential MIMO channel is sufficiently rich in multipath to improve capacity gain with 3 or 4 spatial streams for a $4 \times 4$ MIMO system. Two optimal values of transmitting antenna spacing favor TxBF gain: around $0.5 \lambda$ and $1.25 \lambda$. This result was verified for two different Tx positions and is to be confirmed and generalized to other environments. Tx-BF can improve the transmission range especially when $N_{s s}$ is sufficiently small compared to $N T x$. For future work, we plan to analyze the impact of the equivalent isotropic radiated power (EIRP) constraints imposed in some countries on these conclusions. 


\section{References}

1. H.T. Nguyen, J.B. Andersen, and G.F. Pedersen, "On the Performance of Link Adaptation Techniques in MIMO Systems", Wireless Personal Communications, vol. 35, no. 4, pp. 289-309, Sept. 2007.

2. V. Desai, J.F. Kepler and F.W. Vook, "Field Data showing the Downlink Adaptive Beamforming Gains in an Experimental IEEE 802.16e-2005 OFDMA System", Radio and Wireless Symposium, 2008 IEEE.

3. C. Hermosilla, R.A. Valenzuela, L. Ahumada and R. Feick, "Empirical Comparison of MIMO and Beamforming Schemes for Outdoor-Indoor Scenarios", IEEE Transactions on Wireless Communications, vol. 8, no. 3, pp.1139-1143, March 2009.

4. G.K. Tran, N.D. Dao, K. Sakaguchi, K. Araki, H. Iwai, T. Sakata, K. Ogawa, "Performance Analysis of MIMO-OFDM Systems using Indoor Wideband MIMO Channel Measurement Data", Vehicular Technology Conference, VTC 2006 Spring, IEEE 63 ${ }^{\text {rd }}$, vol. 6, pp. 2868-2872, 7-10 May 2006.

5. H. Nishimoto, Y. Ogawa, T. Nishimura, T. Ohgane, "Measurement-Based Performance Evaluation of MIMO Spatial Multiplexing in a Multipath-Rich Indoor Environment", Antennas and Propagation, IEEE Transactions on, vol. 55, no. 12, pp. 3677 - 3689, Dec. 2007.

6. C. Hermosilla, R.A. Valenzuela, L. Ahumada and R. Feick, "Empirical Comparison of MIMO and Beamforming Schemes", IEEE International Conference on Communications, 2008. ICC '08, pp. 4226-4229, 19-23 May 2008.

7. B. Friedlander and S. Scherzer, "Beamforming versus Transmit Diversity in the Downlink of a Cellular Communications System", IEEE Transactions on Vehicular Technology, vol. 53, no. 4, pp. 1023-1034, July 2004.

8. H. Busche, A. Vanaev and H. Rohling, "SVD-based MIMO Precoding and Equalization Schemes for Realistic Channel Knowledge: Design Criteria and Performance Evaluation", Wireless Personal Communications, 2009-02-01.

9. G. Lebrun, T. Ying and M. Faulkner, "MIMO Transmission over a Time-Varying Channel using SVD", Global Telecommunications Conference, GLOBECOM 'O2, IEEE, vol. 1, no.1, pp. 414418, 17-21 Nov. 2002.

10. G. Morozov, A. Davydov and A. Papathanassiou, "A Novel Combined CSI Feedback Mechanism to support Multi-user MIMO Beamforming Schemes in TDD-OFDMA Systems", International Congress on Ultra Modern Telecommunications and Control Systems and Workshops (ICUMT), 2010, pp. 896-900, 18-20 Oct. 2010.

11. S. Xiantao Q. Wang, L.J. Cimini, L.J. Greenstein and D.S. Chan, "ICI/ISI-Aware Beamforming for MIMO-OFDM Wireless Systems", IEEE Transactions on Wireless Communications, vol.11, no. 1, pp. 378-385, January 2012.

12. S. Quadri and R.J. Haines, "Industrial Prototyping: A Common Architecture and Case Study of IEEE 802.11n Transmit Beamforming", Vehicular Technology Conference, VTC Spring 2009. IEEE 69th, pp. 1-5, 26-29 April 2009.

13. J.B. Anderson, "Array Gain and Capacity for Known Random Channels with Multiple Element Arrays at Both Ends", IEEE Journal on Selected Areas in Communications. 2000, 18(11). 21722178.

14. E. Sengul, E. Akay, and E. Ayanoglu, "Diversity Analysis of Single and Multiple Beamforming," IEEE Transactions on Communications, vol. 54, no. 6, pp. 990-993, Jun. 2006.

15. E. Haddad, N. Malhouroux, P. Pajusco and M. Ney, "Optimization of 3D Ray Tracing for MIMO Indoor Channel”, General Assembly and Scientific Symposium, URSI, pp. 1-4, 13-20 Aug. 2011.

16. G. Tesserault, N. Malhouroux, and P. Pajusco, "Multi-frequencies Characterization of Building Materials: Angular and Polarization Analysis", European Conference on Antennas and Propagation, 2007.

17. D. Senaratne and C. Tellambura, "Generalized Singular Value Decomposition for Coordinated Beamforming in MIMO Systems", Global Telecommunications Conference (GLOBECOM 2010), IEEE, pp. 1-6, 6-10 Dec. 2010.

18. "IEEE Draft Standard for Information Technology - Telecommunications and Information Exchange Between Systems - Local and Metropolitan Area Networks - Specific Requirements - 
Part 11: Wireless LAN Medium Access Control (MAC) and Physical Layer (PHY) Specifications", IEEE P802.11-REVmb/D10.0, August 2011 (Revision of IEEE Std 802.11-2007, as amended by IEEE Std 802.11k-2008, IEEE Std 802.11r-2008, IEEE Std 802.11y-2008, IEEE Std 802.11w-2009 and IEEE Std 802.11n-2009), pp. 1-3026, Aug. 202011.

19. S. Wyne, K. Haneda, S. Ranvier, F. Tufvesson and A.F. Molisch, "Beamforming Effects on Measured mm-Wave Channel Characteristics", IEEE Transactions on Wireless Communications, vol. 10, no. 11, pp. 3553-3559, November 2011.

20. A. Perahia and R. Stacey, "Next Generation Wireless LANs Throughput, Robustness, and Reliability in 802.11n", Cambridge, 2008.

21. X. Jie, D.L. Goeckel and R. Janaswamy, "The Capacity of MIMO Systems with increasing SNR by Electromagnetic Analysis", IEEE Transactions on Wireless Communications, vol. 8, no. 9, pp.4752-4761, September 2009.

22. T.K. Paul and T. Ogunfunmi, "Wireless LAN Comes of Age: Understanding the IEEE 802.11n Amendment", Circuits and Systems Magazine, IEEE, vol. 8, no. 1, pp. 28-54, First Quarter 2008.

23. TGn Channel Models, IEEE Std. 802.11--03/940r4, May, 2004.

24. M. Ozcelik, N. Czink and E. Bonek, "What makes a Good MIMO Channel Model?", Vehicular Technology Conference, VTC-Spring. 2005, IEEE 61 ${ }^{\text {st }}$, vol. 1, pp. 156- 160, 30 May-1 June 2005.

25. D.P. Palomar and M.A. Lagunas, "Joint Transmit-Receive Space-Time Equalization in Spatially Correlated MIMO Channels: a Beamforming Approach", IEEE Journal on Selected Areas in Communications, vol. 21, no. 5, pp. 730- 743, June 2003.

26. T. Rappaport. Wireless Communication: Principles and Practice, IEEE Press, 1996.

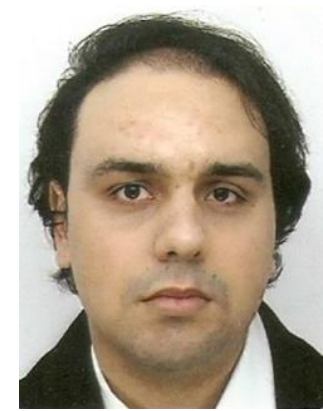

Ali Bouhlel received Engineering degree from ENSEEIHT Toulouse in 2009. In 2010, he joined Orange Labs Belfort and IETR Rennes as Phd student. His current research interests include radio engineering and the effects of indoor MIMO propagation channels on Wireless LANs performance.

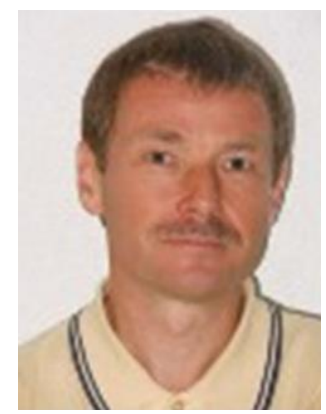

Valéry Guillet received Engineering, and Ph.D. degrees from Telecom Paristech in 1992, 1996 respectively. In 1996, he joined Orange Labs Belfort as a research engineer in the field of propagation channel measurement and modeling from 2.4 to $60 \mathrm{GHz}$ for the future broadband and Wireless LANs design. Then, he has worked as project manager for Wireless LANs planning tool development and WiFi engineering for the Home Network. His current research focuses in the area of radio engineering, performance evaluation and optimization for Wireless LANs and especially for single and multi-user MIMO systems. 


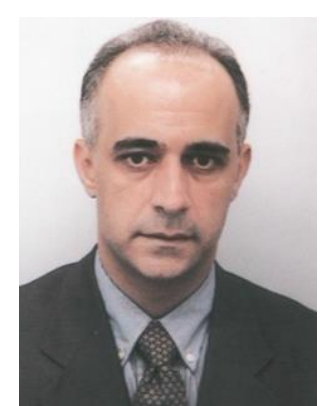

Ghaïs El Zein received the M.S. degree in Electronics, the Ph.D. degree in Telecommunications and Signal Processing, and the Habilitation (H.D.R.) degree in Electronics from the University of Rennes 1 - France, in 1983, 1988 and 1998, respectively. From 1985 to 1987, he was a Lecturer, and from 1990 to 1999, an Associate Professor, in the Department "Communications Systems and Networks" of the "Institut National des Sciences Appliquées de Rennes" (INSA) - France, where he is currently a Professor. His teaching and research interests mainly concern the study of radio wave propagation phenomena and the evaluation of their effects on communication systems. He has authored or co-authored over 220 technical papers in major international journals and conferences, 2 books, 13 book chapters and 4 patents in the areas of communications and radio propagation. He served as a TPC member and symposium chair of several international conferences. From 2001 to 2009, he served as deputy director of the Institute of Electronics and Telecommunications of Rennes (IETR - UMR CNRS 6164). Since 2009, he is the Head of Radio Propagation Team. He is a member of URSI-F and member of the Monitoring and Assessment Committee (CSE) of the "Images et Réseaux" cluster.

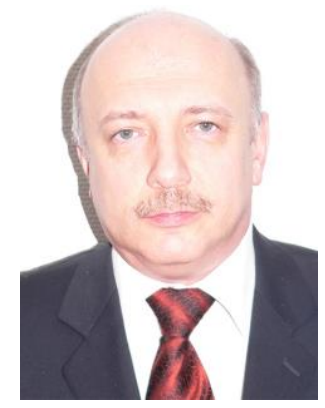

Gheorghe Zaharia received the B.S. degree in electronics and telecommunications from the Polytechnic Institute of Iasi, Romania, in 1981 and the D.E.A. and Ph.D. degrees in telecommunications, signal processing and electronics from the "Institut National des Sciences Appliquées" (INSA) of Rennes, France, in 1991 and 1997, respectively. From 1983 to 1990 he was a Lecturer at the Technical University of Iasi. Between 1983 and 1985 he studied mathematics at the University "Al. I. Cuza" of Iasi. Since 1997, he is Associate Professor in the Department "Communications Systems and Networks", the former "Electronics and Communications Systems" Department, INSA, Rennes. His teaching and research interests mainly concern the study of radio waves propagation, communications systems, information theory, and coding. He is Senior Member IEEE and member of the Romanian Society of Medical Bioengineering. He was member of the Scientific Committee of several international conferences. He has more than 30 journal papers, about 90 papers presented at international conferences and 2 patents. He is also co-author of one book and 3 book chapters. 